\title{
When today's rewards are tomorrow's endowments: The effects of inequality on social competition
}

\author{
Ennio Bilancini ${ }^{\mathrm{a}, *}$, Leonardo Boncinelli ${ }^{\mathrm{b}}$ \\ a IMT School for Advanced Studies Lucca, Piazza S. Francesco 19, Lucca 55100, Italia \\ ${ }^{\mathrm{b}}$ Department of Economics and Management, University of Florence, Via delle Pandette 9, Firenze 50127, Italia
}

\section{A R T I C L E I N F O}

\section{Article history:}

Received 12 February 2019

Revised 15 June 2020

Accepted 23 June 2020

Available online 21 July 2020

\section{JEL classification:}

D44

D62

D63

\section{Keywords:}

Social status

Social competition

Inequality

Redistribution

\begin{abstract}
A B S T R A C T
In this paper we study the role of inequality in a model of repeated social competition where endowments (the resources used in the competition) and rewards (the resources obtained as prizes) are connected because the rewards of today's competition determine the endowments of tomorrow's competition. We find that the harshness of social competition is maximal for an intermediate level of inequality, and that increasing or decreasing inequality beyond that level monotonically mitigates social competition, up to completely eliminating it at the extremes. If social competition is wasteful, utilitarian welfare is maximized in case of equality. A greater uncertainty about the connection between today's rewards and tomorrow's endowments mitigates social competition. Finally, there emerge concerns for others' resources that are downward and not upward, with the value of high social standing which changes non-monotonically in the resources of those with lower standing.
\end{abstract}

(c) 2020 Elsevier B.V. All rights reserved.

\section{Introduction}

The effects of inequality on social competition have recently received increasing attention among economists (Hopkins and Kornienko, 2010; Hopkins, 2012; Bilancini and Boncinelli, 2012; Levy and Razin, 2015; Bhaskar and Hopkins, 2016), yielding a variety of models which, while differing in a number of features, all share a one-period setting. In many realworld applications, however, social competition takes place repeatedly over time, and this is not addressed in previous studies. The present paper attempts to fill this gap: we provide a tractable analytical setup based on a simple auction model of social competition that allows us to study under what conditions inequality fosters or mitigates social competition in an intertemporal setting. In particular, we consider the case in which the rewards of today's competition - the prizes up for grabs - end up forming the endowments of tomorrow's competition - the resources that can be used to outcompete others and win the competition.

We find that the intertemporal connection between endowments and rewards generates an ambiguous overall effect of inequality on social competition. More precisely, we show that there is an intermediate level of inequality that maximizes the harshness of social competition; moreover, increasing or decreasing inequality beyond that point monotonically mitigates social competition, up to completely eliminating it at the extremes. Therefore, if social competition is wasteful, then

\footnotetext{
* Corresponding author.

E-mail addresses: ennio.bilancini@imtlucca.it (E. Bilancini), leonardo.boncinelli@unifi.it (L. Boncinelli).
} 
utilitarian welfare is maximized when inequality is nil, since in this case not only waste is minimized but also marginal utilities are equalized across individuals.

To show the relevance of the intertemporal setting that we study in this paper, we point to the many situations where social competition is not just once and for all, but occurs several times. This is especially true if we consider an individual's entire lifetime: one first competes at school for highest education, then in the job market for best jobs, then again for a better job - and this possibly many times. In many cases the rewards of any of these competitions concur to form the endowments of the subsequent competition. Such connection between endowments and rewards becomes even more evident if we take an intergenerational perspective: parents' success in the social competition determines children's endowments for their competition. The rewards earned by the current generation are passed to the next generation in the form of future endowments, which in turn determine the outcome of future competition, allowing the next generation to earn rewards that will then be passed to the following generation as endowments, and so on and so forth.

Our model represents social competition as a second price auction with two bidders, where the winner only pays, and the payment is equal to second highest bid. Endowments represent resources that can be spent in bidding, while rewards represent the market value of the auctioned social prize. While most of the literature considers all-pay auctions or contests, we have focused on a second price auction mainly for reasons of analytical tractability. Indeed, our model provides an accurate description of social competition only in situations involving refundable bids (as in the sealed bid auction) or revelation of losing bids (as in the English auction); more often, our model can be considered as fictional and heuristic, helping us illustrate in a clear way an intertemporal effect on competitiveness which is at work when the rewards of social competition today end up forming the endowments of social competition tomorrow. ${ }^{1}$

The main intuition behind our results is the following. When today's rewards are tomorrow's endowments, a change in inequality affects both rewards and endowments, but in contrasting directions: a reduction of today's rewards makes the current competition milder, but such greater equality then translates into a greater equality of endowments tomorrow, which in turn makes tomorrow's competition harsher. So, on the whole, the distortions due to social competition may increase or decrease as a result of a greater inequality. The maximum harshness in social competition is obtained for an intermediate level of inequality because the inequality of rewards and the inequality of endowments are to some extent complementary for social competition. To put it simply, if on the one hand there is no incentive to compete for very similar prizes, on the other hand it is hard to get a real competition when the poor competitor has very few resources to burn in the competition.

In more detail, we show that if preferences exhibit non-increasing absolute risk aversion, then the relationship between social competition and inequality is inverted U-shaped. Intuitively, a marginal redistribution changes both the cost of social competition and the benefit of winning the competition relative to losing it. While the first change depends on the steepness of the utility function, the second one depends on its concavity. It can be shown that the latter is smaller than the former if we require that the second derivative changes not more than proportionally with respect to the first derivative, which amounts to assuming a non-increasing coefficient of absolute risk aversion. Under such assumption, the change in social competition due to a marginal redistribution of resources decreases as inequality increases, resulting in a humped shape of the relationship between social competition and inequality. We stress that experimental and empirical evidence is mostly consistent with decreasing absolute risk aversion (Friend and Blume, 1975). ${ }^{2}$

We also explore the case of an uncertain connection between today's rewards and tomorrow's endowments, and in particular we consider an intergenerational model where agents live for two periods and pass their social standing to their descendants (about whom they care as themselves) with positive probability. We show that a smaller inequality when young (old) mitigates social competition when old (young), because it negatively affects the future stream of rewards associated with a high social standing. Further, and perhaps more interestingly, we show that a smaller probability of passing one's current social standing to the next generation mitigates social competition among both young and old individuals because it makes the expected payoffs of descendants less dependent on the outcome of the social competitions of the current generation.

Finally, we apply the instrumental approach to social preferences (Postlewaite, 1998) in order to investigate which kind of concerns for others emerge in equilibrium. We find that an individual with a low social standing (the loser of the social competition) has no instrumental concerns for others, of any kind. By contrast, an individual with high social standing (the winner of the social competition) is instrumentally concerned about the endowment of those with lower standing, and this in a way that depends non-monotonically on the endowment's level associated with low social standing: if the endowment is sufficiently small (large) then an even smaller (larger) endowment is preferred.

The paper is organized as follows. In Section 2 we survey and discuss the related literature. In Section 3 we describe the model and the equilibrium concept used in the following analysis. In Section 4 we provide the main results regarding the relationship between inequality and social competition. In Section 5 we discuss applications and extensions of the analysis. In Section 6 we provide final remarks. All proofs of the propositions provided in the paper are relegated to the Appendix.

\footnotetext{
1 To check the robustness of our results, in Appendix B, we provide a formal analysis of a signaling variant of the model, where we show the equivalence between the amount of resources spent in signaling and the price paid in the second price auction.

2 An example of a decreasing absolute risk aversion utility function is $u(c)=\log (c)$, and an example of a constant absolute risk aversion utility function is $u(c)=1-e^{\alpha c}$.
} 


\section{Literature review}

Social competition often generates pervasive externalities that can affect deeply economic behavior (see Truyts, 2010, and references therein). Such externalities can lead to severe market inefficiencies (Frank, 2008; Aronsson and JohanssonStenman, 2010), and even shape preferences for public policy measures possibly aimed at correcting those inefficient outcomes (see Gallice, 2018, and references therein). At the same time, social competition can help internalize previously existing externalities, thus being potentially beneficial (Hopkins, 2012; Bhaskar and Hopkins, 2016).

One important issue is how inequality affects the harshness of the social competition, and hence how it affects the related externalities. Hopkins and Kornienko (2010) argue that it is crucial to distinguish between two dimensions of inequality: the inequality of endowments, i.e., the social and economic resources employed in the social competition, and the inequality of rewards, i.e., the social and economic resources obtained as the prize of competition. In particular, Hopkins and Kornienko (2010) show that less inequality in endowments fosters social competition (as obtained in Hopkins and Kornienko, 2004), while less inequality in rewards mitigates social competition. So, more inequality in endowments is a substitute for more equality in rewards when the objective is to mitigate the distortions induced by social competition. Notably, when endowments and rewards can be modified independently from each other, we are left with a simple and very effective policy recipe: if the distortions due to social competition are undesired, then a policy-maker should reduce the inequality of rewards, while increasing the inequality of endowments.

Sometimes, however, endowments and rewards are inherently related to each other, with the result that they cannot be modified independently. In such cases, a change in the inequality of endowments may be necessarily associated with a change in the inequality of rewards, and the overall effect is in general indeterminate, as shown in Bilancini and Boncinelli (2012). This is the case, for instance, when endowments are income potentials and the size of rewards depends on the income actually earned, not on the income potential (Bilancini and Boncinelli, 2019). In the present paper we look at a different reason why endowments and rewards are connected: the rewards of today's social competition can determine tomorrow's endowments for the next social competition.

In a setup with ordinal concerns for social status, that are induced by assortative matching in the marriage market, Levy and Razin (2015) find that extreme distributions can be actually preferred by the majority of the population and become attractors: if the distribution is sufficiently equal, then agents ask for more equality, while if the distribution is sufficiently unequal, then agents ask for less equality. This result stems from the fact that a more equal distribution of endowments generates stronger social competition which in turn generates greater sorting costs.

This is in part related to what has been found by Ireland (1998) and Corneo (2002) regarding the role of pre-tax wage inequality on redistributive labor taxes: ${ }^{3}$ when inequality is high to begin with, then redistributing income through labor income taxes increases social competition, while it mitigates social competition when inequality is initially low. The underlying mechanism of these results is different from the one considered in the present paper, and actually it works only when concerns for social status are ordinal (i.e., rewards are exogenously fixed; see Bilancini and Boncinelli, 2019): labor income taxes always reduce inequality in endowments, which would foster social competition, but at the same time they also reduce the relative gains of working over not working; if the first effect is not too strong, which happens when pre-tax wage inequality is low (and hence social competition is already strong), then a labor income tax mitigates the social competition by disincentivizing work.

Finally, by focusing on the unique symmetric Markov Perfect Equilibrium in weakly undominated strategies we find that social competition does not translate into social mobility, no matter how harsh the competition is. This is due to the fact that along the equilibrium path higher types are always successful in deterring the overtaking by lower types. Such lack of social mobility is in line with well established results for dynastic models with social competition, as pioneered by Cole et al. (1992). Similar results are also found by Corneo and Jeanne (1999) in a two-class context and by Corneo and Jeanne (2001) for a setup with a continuum of types.

\section{Model}

We consider the following two-player intertemporal setting, with discrete time and infinite horizon, which is intended to be representative of the social competition. ${ }^{4}$ The crucial feature that we introduce here is that the resources that are earned at one time become the endowments at the following time.

We restrict attention to a case where resources are constant over time. At each time the distribution of resources is such that $R_{H}$ resources are for one player, $R_{L}$ resources are for the other player, with $R_{H}>R_{L}$. Players compete with each other in a complete information second-price auction. The player who makes the highest offer wins the competition, paying the offer made by the other player and obtaining $R_{H}$ resources for the future, while the other player pays nothing and obtains $R_{L}$ resources for the future.

\footnotetext{
3 See also Aronsson and Johansson-Stenman (2013) for the case where also leisure concurs to determine one's social standing.

4 The two-player assumption allows us to develop a simple model. We note that the model could be easily adapted to have $2 N$ players, $N$ of them corresponding to one player, the remaining $N$ corresponding to the other player. Also, the modeling approach in Hopkins and Kornienko (2004) might be used to explore the case with a continuum of players.
} 
We assume that the overall utility for a player is given by the discounted sum of utilities over time. The utility accruing to a player at each time is generated by the net resources at that time after the offer is paid (which happens only if the competition is won). We denote the time-by-time utility function with $U$ and the discount factor with $\delta$. We assume that $U$ is a twice differentiable and strictly concave function, and we denote with $U^{\prime}$ its first order derivative. Furthermore, we assume that $\lim _{R_{z} \rightarrow 0} U\left(R_{z}\right)=-\infty,,^{5}$ and that the coefficient of absolute risk aversion $-U^{\prime \prime}\left(R_{z}\right) / U^{\prime}\left(R_{z}\right)$ is never increasing in $R_{z}$.

As a solution concept, we consider the Markov Perfect Equilibrium (Maskin and Tirole, 2001). Basically, a Markov Perfect Equilibrium (MPE) is a subgame perfect equilibrium in which all players use Markov strategies. A Markov strategy is a strategy that is function only of the current state, with the current state being a description of all past play of the game which is payoff relevant for future play. In the setting under consideration, a state is simply the indication of having won or lost the last round of competition. Therefore, a Markov strategy can be written as $x:\left\{R_{H}, R_{L}\right\} \rightarrow \mathbb{R}_{+}$; as a shortcut, we write $\left(x_{H}, x_{L}\right)$, with $x_{H}:=x\left(R_{H}\right)$ and $x_{L}:=x\left(R_{L}\right)$.

In this infinite horizon setting, even if we restrict attention to symmetric profiles (which are typically considered as more focal), a multiplicity of Markov Perfect Equilibria exist, including profiles where winners of the social competition always pay nothing, and profiles where the players alternate in winning the competition. As an example of the former case, consider a profile where $x_{L}=0$ and $x_{H}$ is large enough to discourage the player with $R_{L}$ resources to overbid, but not so high to make a zero bid profitable for the player with $R_{H}$ resources. We note that the previous profile would remain a Markov Perfect Equilibrium for any positive $x_{L}$ that is not so high to make losing the competition the preferred choice by the player with $R_{H}$ resources; moreover, even admitting mixed strategies, we note that any strategy randomizing between the possible values of $x_{L}$ would work the same. As an example of a profile where the players alternate in winning the competition, consider $x_{L}>x_{H} \geq 0$, with $x_{L}$ high enough for the player with initially $R_{H}$ resources not to prefer having $R_{H}-x_{L}$ resources at any time over alternating period by period between $R_{H}$ and $R_{L}-x_{L}$ net resources, but not so high to be unaffordable by the player with $R_{L}$ resources. However, all the Markov Perfect Equilibria above sketched do make use of weakly dominated strategies.

Actually, there exists only one symmetric Markov Perfect Equilibrium in weakly undominated strategies, as formally stated in Proposition 1. To get the intuition, consider a profile with $x_{L}=0$ and $x_{H}$ high enough, so that the player with $R_{L}$ resources is indifferent to any bid lower than $x_{H}$, and strictly worse in case of a bid higher than $x_{H}$. We note that, for the player with $R_{L}$ resources, any positive bid $x^{\prime}$ that does not exceed his willingness to pay for the competitive prize weakly dominates the current bid $x_{L}=0$ : indeed, the same payoff is obtained whenever $x_{H}>x^{\prime}$, while a higher payoff is obtained when $x_{H} \leq x^{\prime}$. Such a profile would hence be refined away if we restrict attention to equilibria in weakly undominated strategies.

Proposition 1. The unique symmetric Markov Perfect Equilibrium in weakly undominated strategies is made of strategy ( $x_{H}^{*}, x_{L}^{*}$ ), where $x_{H}^{*}$ and $x_{L}^{*}$ are the unique solutions to:

$$
\begin{aligned}
& U\left(R_{H}-x_{H}^{*}\right)+\sum_{t=1}^{\infty} \delta^{t} U\left(R_{H}-x_{L}^{*}\right)=U\left(R_{H}\right)+\sum_{t=1}^{\infty} \delta^{t} U\left(R_{L}\right) ; \\
& U\left(R_{L}-x_{L}^{*}\right)+\sum_{t=1}^{\infty} \delta^{t} U\left(R_{H}-x_{L}^{*}\right)=U\left(R_{L}\right)+\sum_{t=1}^{\infty} \delta^{t} U\left(R_{L}\right) .
\end{aligned}
$$

In words, the unique symmetric Markov Perfect Equilibrium in weakly undominated strategies is the profile where each player's offer is equal to his willingness to pay for being always rich in the future instead of always poor, i.e., having at any future time $R_{H}-x_{L}^{*}$ net resources instead of $R_{L}$ resources. We observe that $x_{H}^{*}>x_{L}^{*}$, due to the strict concavity of function $U$, hence social mobility never occurs in $\left(x_{H}^{*}, x_{L}^{*}\right)$ : starting the game with $R_{H}$ resources allows a player to earn $R_{H}-x_{L}^{*}$ resources instead of $R_{L}$ resources at any following time. Since, in equilibrium, we have that $x_{H}^{*}>x_{L}^{*}$, the winner of the social competition ends up paying $x_{L}^{*}$, which can then be used as a measure of social waste.

In the following analysis we focus on the unique symmetric Markov Perfect Equilibrium in weakly undominated strategies, which we sometimes refer to simply as equilibrium.

\section{Results}

We define a simple measure of the degree of inequality, $I$, as the difference between $R_{H}$ and $R_{L}$, i.e., $I=R_{H}-R_{L}$. For convenience of exposition, we also define $R=R_{H}+R_{L}$. Most of the results of this paper involve comparative statics on $I$. Since we are interested in the amount of resources that are burnt in equilibrium, we focus on $x_{L}^{*}$ referring to it as harshness of social competition, and we simplify the notation by denoting $x_{\perp}^{*}$ simply with $x^{*}$. We remark that $x^{*}$ can be seen as a function of $R_{L}$. In the following section we will deal with the effects of inequality on social competition, essentially by studying how a change in $R_{L}$, as a consequence of a change in $I$, affects $x^{*}$.

\footnotetext{
${ }^{5}$ This last assumption ensures that the optimal $x_{L}$ is lower than $R_{L}$, so that an interior solution always exists.
} 


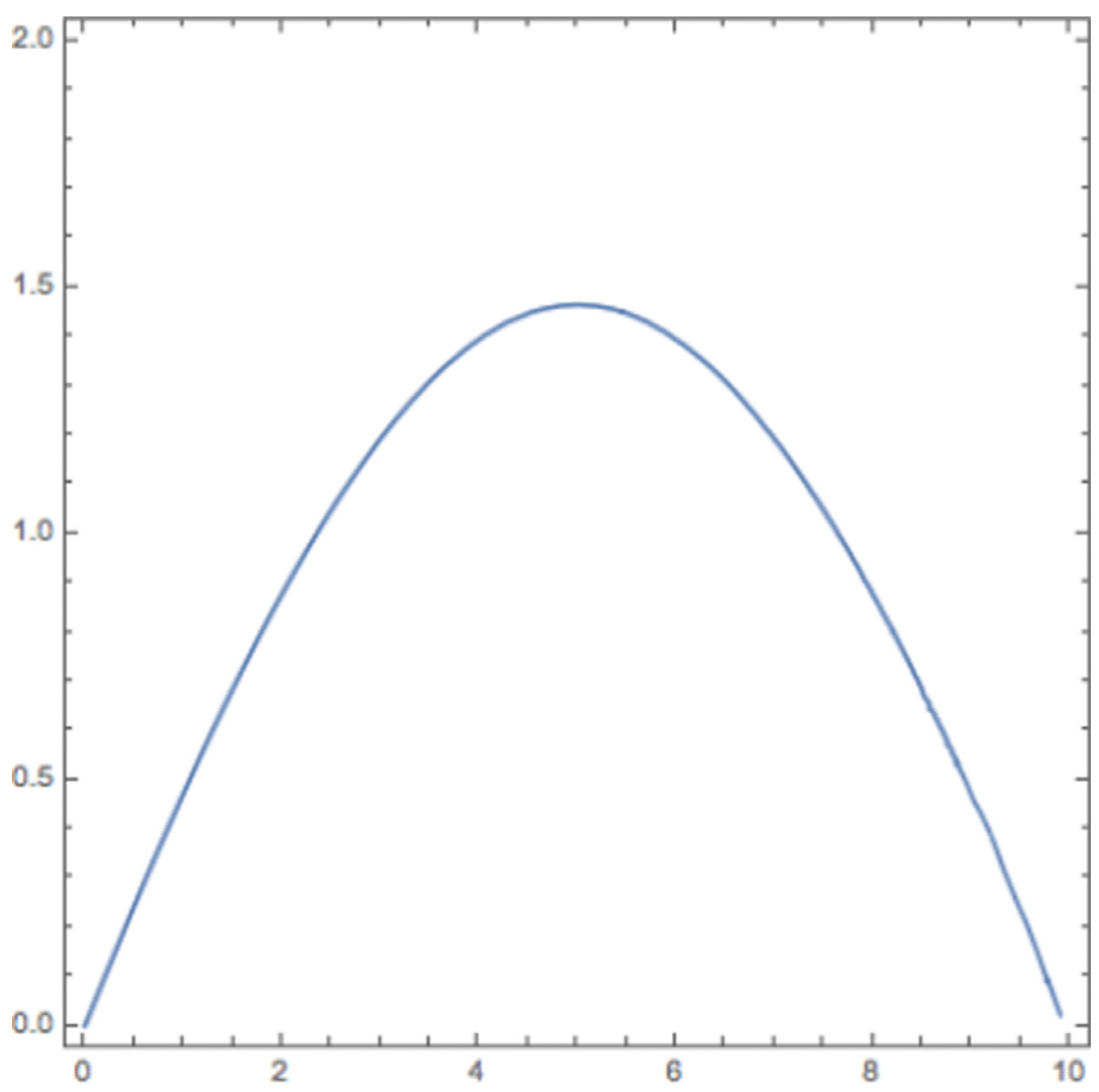

Fig. 1. Plot of $x^{*}$ (vertical axis) as a function of $I$ (horizontal axis), when $U(\cdot)=\ln (\cdot), R=10$, and $\delta=0.5$. In this case we can obtain an explicit function for $x^{*}$, which turns out to be $x^{*}=\frac{10-\sqrt{100-20 I+2 l^{2}}}{2}$.

\subsection{Inequality and social competition}

We now turn our attention to the main question of this paper: which is the overall effect on social competition of an increase in equality of the distribution of resources?

Proposition 2. There exists a threshold degree of inequality $\hat{I}$ such that a marginal redistribution of resources makes $x^{*}$ smaller for $I<\hat{I}$, and larger for $I>\hat{I}$. Moreover, $x^{*}$ tends to 0 both when $I$ tends to 0 and when $I$ tends to $R$.

Proposition 2 states that the harshness of social competition decreases as a result of a marginal redistribution only when inequality is low (i.e., lower than $\hat{I}$ ), while it increases when inequality is high (i.e., higher than $\hat{I}$ ). Intuitively, the net effect of reducing the inequality in both endowments and rewards depends on which of the two has a stronger effect on social competition, since they push in opposite directions. In the light of this we can say that a high initial inequality makes the endowment effect stronger than the reward effect, and vice versa for a low initial inequality. At the threshold inequality $\hat{I}$ the two effects exactly compensate with each other. Fig. 1 illustrates.

\subsection{Welfare}

In the following we provide a simple discussion on the relationship between inequality and social welfare as measured by the total sum of present value utilities.

Preliminarily, we define:

$$
\begin{aligned}
& V_{H}=\sum_{t=0}^{\infty} \delta^{t} U\left(R_{H}-x^{*}\right) ; \\
& V_{L}=\sum_{t=0}^{\infty} \delta^{t} U\left(R_{L}\right) ;
\end{aligned}
$$


i.e., $V_{H}$ and $V_{L}$ are the present value of the stream of utilities earned by, respectively, the individual with high resources and the individual with low resources.

Also, we define social welfare as:

$$
W=V_{H}+V_{L}
$$

i.e., $W$ is the sum of the discounted stream of utilities for the two individuals. We observe that $W=(1 /(1-\delta))\left(U\left(R_{H}-\right.\right.$ $\left.\left.x^{*}\right)+U\left(R_{L}\right)\right)$.

The inspection of $W$ reveals that a smaller $x^{*}$ enhances social welfare, in that it increases $V_{H}$. However, $x^{*}$ varies only in reaction to changes in the degree of inequality, which also affects $W$. Proposition 3 assesses the desirability in terms of social welfare of moving towards full equality:

Proposition 3. Social welfare tends to be maximum when inequality tends to zero.

Intuitively, there are two possible sources of welfare reduction. One is the waste in the social competition. The other is the inequality in the distribution of resources, because of the strict concavity of the utility function. When the degree of inequality tends to zero, this second source of welfare reduction is clearly neutralized; moreover, we know from Proposition 2 that also the waste in the social competition tends to disappear, so that moving towards full equality allows to approach the highest welfare. Note that also moving towards full inequality - when all resources are given to one individual - allows to minimize the waste in the social competition, but it leads to an extremely severe loss of welfare due to the unequal split of resources.

\subsection{Discount factor and social competition}

The next result regards the impact of an increase in the discount factor on the amount of resources spent in the social competition.

Proposition 4. An increase in the discount factor $\delta$ yields an increase in $x^{*}$ for any degree of inequality $I$.

The intuition behind the result in Proposition 4 is straightforward. When the discount factor increases, the present value of the future stream of utilities gets larger, and this translates into a harsher competition - that is, more resources are burnt in the social competition. Fig. 2 illustrates. It is worth to stress that this result depends crucially on the fact that tomorrow's endowments are todays' rewards. If, instead, tomorrow's social competition is independent of today's social competition then the effect of the discount factor on waste is ambiguous, and can possibly be nil; this holds also if individuals can save today's resources for tomorrow's competition (Corneo and Jeanne, 1998) affecting the relative harshness of competition between today and tomorrow (of course this does not hold when social competition is only today, and not tomorrow, as in Hopkins and Kornienko, 2006).

\section{Discussion}

Beyond the analysis provided in Section 4, the proposed model is simple and tractable enough to be naturally extended to investigate related issues. We maintain the focus on the equilibrium where, at each time, the resources spent by the individual with high resources in the social competition make the individual with low resources indifferent between winning the social competition and paying the offered amount of resources, and losing it.

\subsection{Uncertain rewards}

We consider the case where the link between today's rewards and tomorrow's endowments is not certain. To fix ideas and provide a concrete framing for our analysis we interpret the connection between rewards and endowments as intergenerational transmission: when young, individuals compete for social standing using as endowments the inherited resources and then when old they obtain as rewards new resources which they can consume or employ in a second social competition whose rewards will be the endowments of the next generation. Agents care about their descendants as they care about themselves, with appropriate time discount. The uncertainty of the link between rewards and endowments is introduced by assuming that there is a probability $p$ that the endowments are allocated randomly when individuals are young, in practice not taking into account the result of the social competition of the last generation of old individuals.

Formally, the present utility of an individual who is $H$ when young and manages to win the social competition both when young and when old is given by

$$
V_{H}=U\left(R_{H}^{y}-x_{H}^{y}\right)+\delta U\left(R_{H}^{o}-x_{H}^{o}\right)+p \delta^{2} \bar{V}+(1-p) \delta^{2} V_{H},
$$

while the present utility of an individual who is $L$ when young and loses both social competitions is given by

$$
V_{L}=U\left(R_{L}^{y}-x_{L}^{y}\right)+\delta U\left(R_{L}^{o}-x_{L}^{o}\right)+p \delta^{2} \bar{V}+(1-p) \delta^{2} V_{L},
$$

where $R_{z}^{y}$ and $R_{z}^{o}$ denote, respectively, $z$ 's resources when young and $z$ 's resources when old, and $\bar{V}=\frac{1}{2} V_{H}+\frac{1}{2} V_{L}$ is the expected value of a random allocation of endowments. 


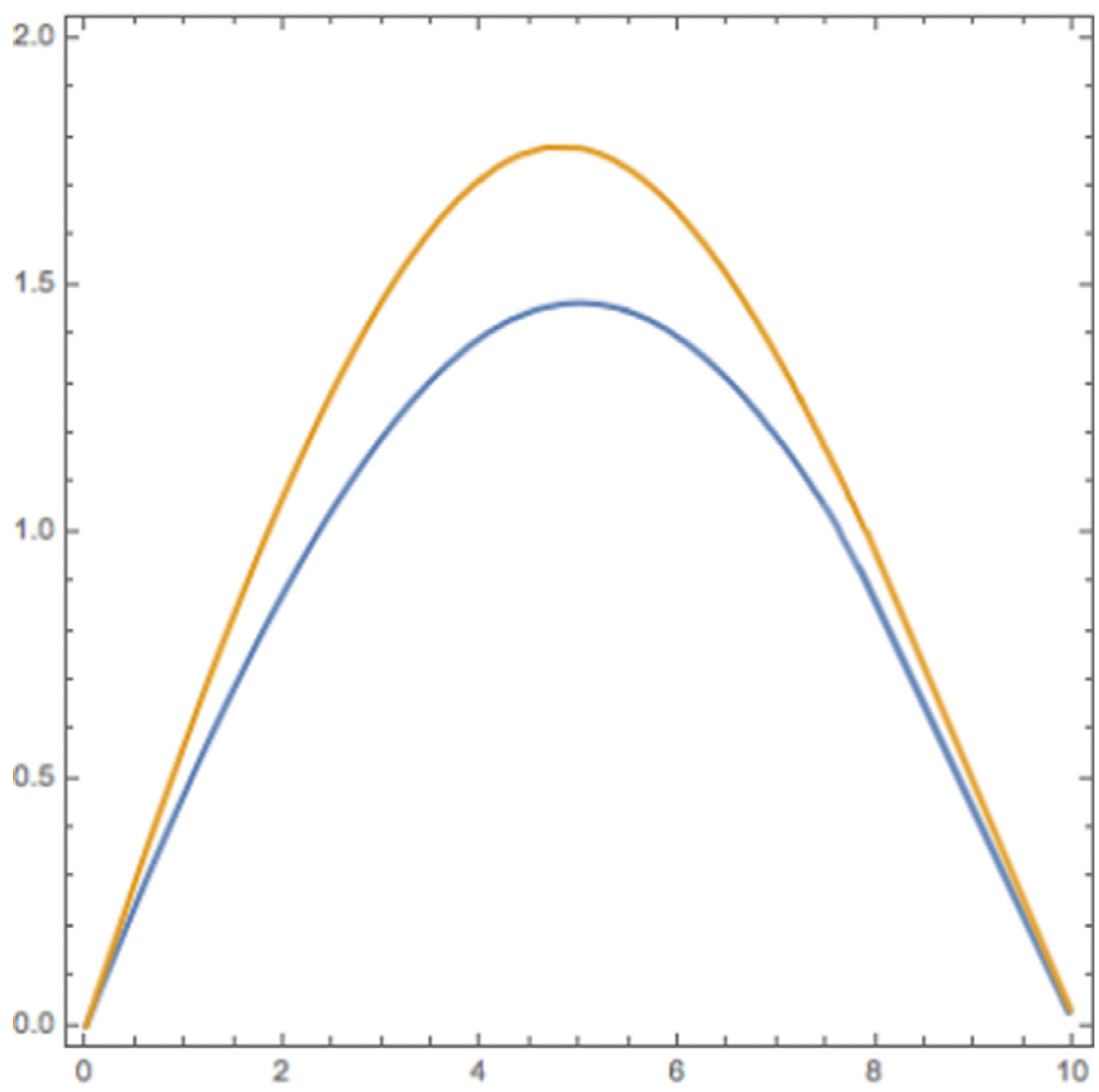

Fig. 2. Plot of $x^{*}$ (vertical axis) as a function of $I$ (horizontal axis), when $U(\cdot)=\ln (\cdot)$ and $R=10$. The blue curve is for $\delta=0.5$ while the yellow curve is for $\delta=0.6$. (For interpretation of the references to colour in this figure legend, the reader is referred to the web version of this article.)

We further define two distinct measures of the degree of inequality: $I_{y}$ for the inequality when young and $I_{o}$ for the inequality when old, respectively given by $I_{y}=R_{H}^{y}-R_{L}^{y}$ and $I_{o}=R_{H}^{o}-R_{L}^{o}$. Also, we denote with $x_{y}^{*}$ and $x_{o}^{*}$ the amount of resources spent for social competition when young and when old, respectively.

To simplify the exposition, we directly focus on the conditions identifying the values of $x_{y}^{*}$ and $x_{0}^{*}$ (which in the Appendix are shown to exist and be unique):

$$
\begin{gathered}
U\left(R_{L}^{o}-x_{o}^{*}\right)+\frac{(1-p) \delta}{1-(1-p) \delta^{2}}\left[U\left(R_{H}^{y}-x_{y}^{*}\right)+\delta U\left(R_{H}^{o}-x_{o}^{*}\right)\right]=U\left(R_{L}^{o}\right)+\frac{(1-p) \delta}{1-(1-p) \delta^{2}}\left[U\left(R_{L}^{y}\right)+\delta U\left(R_{L}^{o}\right)\right] ; \\
U\left(R_{L}^{y}-x_{y}^{*}\right)+\delta U\left(R_{H}^{o}-x_{o}^{*}\right)+\frac{(1-p) \delta^{2}}{1-(1-p) \delta^{2}}\left[U\left(R_{H}^{y}-x_{y}^{*}\right)+\delta U\left(R_{H}^{o}-x_{o}^{*}\right)\right] \\
=U\left(R_{L}^{y}\right)+\delta U\left(R_{L}^{o}\right)+\frac{(1-p) \delta^{2}}{1-(1-p) \delta^{2}}\left[U\left(R_{L}^{y}\right)+\delta U\left(R_{L}^{o}\right)\right] ;
\end{gathered}
$$

where (8) requires an old individual who is $L$ to be indifferent between having $H$ descendants and having $L$ descendants, while (9) requires a young individual who is $L$ to be indifferent between becoming $H$ when old and having $H$ descendants and remaining $L$ when old and having $L$ descendants.

We now analyze the impact of $I_{y}$ and $I_{0}$ on $x_{y}^{*}$ and $x_{0}^{*}$, hence shedding some light on how social competition is affected by inequality.

Proposition 5. A smaller degree of inequality $I_{y}$ leads to a smaller $x_{0}^{*}$ while a smaller degree of inequality $I_{0}$ leads to a smaller $x_{y}^{*}$.

The intuition of Proposition 5 is the following: a smaller inequality in the distribution of resources when young (old) in period $t$ translates into a smaller inequality of future rewards from the perspective of an old (young) individual in period $t+1$. This is because such a redistribution has no impact on resources in period $t+1$, while it affects the resources at $t+2$ 
and later periods, and hence it affects the present value of winning the social competition. By the same token, we observe that both the effect of a smaller $I_{y}$ on $x_{y}^{*}$ and the effect of a smaller $I_{o}$ on $x_{0}^{*}$ are ambiguous and depend on the current level of both $I_{y}$ and $I_{o}$, in line with what found in Section 4. Overall, considering that $I_{y}$ and $I_{0}$ can be jointly reduced, these results suggest that redistributing resources tends to mitigate social competition.

Moreover, we can study what is the effect of a greater uncertainty on the harshness of the social competition.

Proposition 6. A greater $p$ leads to a smaller $x_{0}^{*}$ and a smaller $x_{y}^{*}$.

The intuition for the impact of $p$ on social competition is as follows: a greater uncertainty weakens the link between one's social standing and that of his descendants, which in turn reduces the future rewards of the social competition, and this is true for both young and old individuals. Finally, note that this effect is typically stronger for old individuals as the competition of young individuals for their resources when old is unaffected.

In Section 4.3 we have seen that a greater importance of future resources induces a harsher social competition today, possibly making it undesirable to have individuals who care a lot about the very long run. Proposition 6 suggests that this could be counteracted by increasing the uncertainty, because this reduces the advantages of winning today's social competition without affecting the relative importance of future consumption.

\subsection{The shape of instrumental concerns for others}

Cole et al. (1992) have shown that social competition can induce instrumental concerns for others, and in particular concerns for social status. ${ }^{6}$ However, not all instrumental concerns for status are the same. In some cases individuals are concerned only about their rank in the distribution of the relevant attributes - e.g., resources in this paper. We call these ordinal status concerns: agents only care about their rank in the distribution, since only changes in the rank determine changes in the allocation of rewards. In other cases individuals are not only concerned with their rank, but also with other features of the distribution, such as for instance the difference between one's resources and others' resources. We call these cardinal status concerns: agents care about some non-ordinal feature of the distribution of resources because it affects the size of net rewards obtained. Further, we distinguish cardinal status concerns that are upward - when the value of status is reduced by an increase in the resources held by individuals of higher rank - from cardinal status concerns that are downward - when the value of status is reduced by an increase in the resources held by individuals of lower rank. ${ }^{7}$

Importantly, social competition needs not necessarily induce instrumental concerns for others that take the form of concerns for status, but it can lead to the emergence of a variety of concerns for others: conformism, inequity aversion, snobbism, and other (Postlewaite, 1998). To see what is the actual shape of concerns for others when today's rewards of the social competition affect tomorrow's endowments for the next competition, we follow the instrumental approach to social preferences (Postlewaite, 1998; Bilancini and Boncinelli, 2014): utilities are considered in the reduced form resulting from the equilibrium of the social game. For this analysis we consider the model of Section 3, taking the present value of the entire stream of rewards determined by today's social competition as the value of today's social standing. In the absence of social mobility, as it happens in the equilibrium we focus on, we have that:

$$
\begin{aligned}
& S_{H}=\sum_{t=1}^{\infty} \delta^{t} U\left(R_{H}-x^{*}\right) ; \\
& S_{L}=\sum_{t=1}^{\infty} \delta^{t} U\left(R_{L}\right) ;
\end{aligned}
$$

where $S_{H}$ is the overall present value of winning today's competition while $S_{L}$ is the overall present value of losing it.

To derive the shape of instrumental concerns for others we look at how $S_{H}$ is affected by a change in $R_{L}$ taking $R_{H}$ as fixed and, similarly, at how $S_{L}$ is affected by a change in $R_{H}$ taking $R_{L}$ as fixed. The following proposition summarizes the results in this regard.

Proposition 7. A marginal increase in $R_{H}$ has no effect on $S_{L}$. Moreover, for any given $R_{H}$, there exists a threshold $\tilde{R}_{L}$ such that:

(i) for $R_{L} \in\left(0, \tilde{R}_{L}\right)$ a marginal increase in $R_{L}$ reduces $S_{H}$;

(ii) for $R_{L} \in\left(\tilde{R}_{L}, R_{H}\right)$ a marginal increase in $R_{L}$ increases $S_{H}$.

Proposition 7 clarifies that the future stream of utilities accruing to an individual whose current social standing is $L$ is not affected at all by what happens to $R_{H}$. This means that an individual who has $R_{L}$ resources today has no instrumental concerns for others, of any kind. By contrast, the future stream of utilities accruing to an individual whose current social standing is $H$ is affected by changes in $R_{L}$, meaning that the individual who has $R_{H}$ resources today does show instrumental

\footnotetext{
${ }^{6}$ Concerns are instrumental when they are the result of an underlying social game that generates them, and do not derive directly from preferences (Postlewaite, 1998).

7 See the taxonomy discussed in Bilancini and Boncinelli (2014) for a formal definition of the shapes of status concerns when the relevant attributes are distributed over a continuum.
} 
concerns for others. Interestingly, the actual shape of such concerns depends on the distribution of resources: for low enough $R_{L}$ there is an instrumental preference for even lower $R_{L}$, while for high $R_{L}$ there is an instrumental preference for even higher $R_{L}$. Hence, for low $R_{L}$ we have instrumental concerns for others that resemble those for social status, and in particular cardinal concerns that are downward but not upward. Instead, for high $R_{L}$ we have instrumental concerns for others that resemble kindness towards the less endowed, and only towards them.

The intuition for these results is the following. In equilibrium, an individual with $R_{L}$ resources today wins neither today's social competition nor any of the future ones and so he never pays or gains anything if $R_{H}$ changes. As a consequence, he has no instrumental concerns over $R_{H}$. On the contrary an individual with $R_{H}$ resources today wins both today's social competition and all future ones and so he pays $x^{*}$ in each period; this generates an instrumental concern over $R_{L}$ and its shape depends on how $R_{L}$ affects $x^{*}$. Two cases are possible, since an increase in $R_{L}$ triggers two opposite effects: a smaller cost to compete (a smaller disutility of $x$ due to decreasing marginal utility) and a smaller incentive to compete (a smaller net gain of winning the competition due to a smaller difference in rewards). For low $R_{L}$ the first effect dominates, while for high $R_{H}$ the second effect dominates.

\section{Conclusions}

In this paper we have explored how inequality impacts the harshness of social competition when the rewards of the competition that takes place today also determine the endowments of the competition that will take place tomorrow. Overall, our findings suggest that social competition is fiercest for an intermediate level of inequality. This in turn implies that more equality can be desirable from a utilitarian perspective, due to the lower marginal utility from resources of richer individuals.

From a methodological standpoint, our analysis shows that it is possible to provide a specific microfoundation of the connection between endowments and rewards in social competitions. However, it is fair to say that endowments and rewards might be connected through channels other than the one considered here, and with possibly completely different consequences in terms of the relationship between inequality and intensity of the social competition. For instance, social competition might be two-sided as in Bhaskar and Hopkins (2016) and rewards may accrue from the amount of endowments that one's mate has burned in the competition. Another possibility is that the dissipation of endowments in the social competition produces an increase in productivity that in turn increases future rewards, though possibly in a differentiated way across the social ladder (Gallice and Grillo, 2019b), or that social status is in part exogenous to the social competition depending on a class structure (Corneo and Gruner, 2000; Gallice and Grillo, 2019a). One might also consider social conflict as part of the story, allowing endowments to serve as means for both the social competition and the social struggle, where the social competition allocates the rewards while the social struggle determines how much they are differentiated. We believe that such and similar alternative sources of interdependence between rewards and endowments may be worth exploration. Finally, it may be worth investigating models with more than just two players (or social classes), for instance by including an intermediate player (representing the middle class); such a richer setting would allow to investigate the effects of policy interventions affecting the distributions of resources in different, yet natural, ways such as moving the intermediate reward closer to the low (or high) reward.

\section{Appendix A. Proofs}

\section{Proof of Proposition 1}

Suppose that $x_{H}^{*}>x_{L}^{*}$. Here having $R_{H}$ resources allows to win the competition and obtain $R_{H}$ resources to start with in the next round of competition, while having $R_{L}$ resources leads to lose the competition and obtain $R_{L}$ resources. We can write the present discounted value of the resulting streams of payoffs as $V_{H}=U\left(R_{H}-x_{L}^{*}\right)+\delta U\left(R_{H}-x_{L}^{*}\right)+\delta^{2} U\left(R_{H}-x_{L}^{*}\right)+$ $\ldots=\frac{U\left(R_{H}-x_{L}^{*}\right)}{1-\delta}$ and $V_{L}=U\left(R_{L}\right)+\delta U\left(R_{L}\right)+\delta^{2} U\left(R_{L}\right)+\ldots=\frac{U\left(R_{L}\right)}{1-\delta}$.

As a consequence, the present discounted value of the streams of payoffs which are triggered by winning and losing today's competition are equal to, respectively, $\delta V_{H}$ and $\delta V_{L}$. Suppose that $x_{L}^{*}<R_{H}-R_{L}$; hence, $\delta V_{H}>\delta V_{L}$. It is immediate to conclude that a strategy would be weakly dominated if the bid is different from the willingness to pay for obtaining $\delta V_{H}$ instead of $\delta V_{L}$. Therefore:

$$
\begin{gathered}
U\left(R_{H}-x_{H}^{*}\right)+\delta V_{H}=U\left(R_{H}\right)+\delta V_{L} ; \\
U\left(R_{L}-x_{L}^{*}\right)+\delta V_{H}=U\left(R_{L}\right)+\delta V_{L} .
\end{gathered}
$$

It is easy to verify that the unique solutions to the above equations indeed satisfy the assumptions that $x_{H}^{*}>x_{L}^{*}$ and $x_{L}^{*}<R_{H}-R_{L}$. Hence, we have found the unique pair $\left(x_{H}^{*}, x_{L}^{*}\right)$ which gives rise to a symmetric Markov Perfect Equilibrium in weakly undominated strategies, when $x_{H}^{*}>x_{L}^{*}$ and $x_{L}^{*}<R_{H}-R_{L}$. In the following, we consider the other cases.

While maintaining that $x_{H}^{*}>x_{L}^{*}$, we now assume that $x_{L}^{*}>R_{H}-R_{L}$. In this case, $\delta V_{H}<\delta V_{L}$. Therefore, the optimal bid at time $t=0$ is zero independently from the amount of resources possessed, which is against the assumption $x_{H}^{*}>x_{L}^{*}$. 
Suppose that $x_{H}^{*}<x_{L}^{*}$. This means that the competition is won when possessing $R_{L}$ resources, and lost when possessing $R_{H}$ resources. The present value of the resulting streams of net resources are $V_{H}=U\left(R_{H}\right)+\delta U\left(R_{L}-x_{H}^{*}\right)+\delta^{2} U\left(R_{H}\right)+\ldots=$ $\frac{U\left(R_{H}\right)+\delta U\left(R_{L}-x_{H}^{*}\right)}{1-\delta^{2}}$ and $V_{L}=\frac{U\left(R_{L}-x_{H}^{*}\right)+\delta U\left(R_{H}\right)}{1-\delta^{2}}$. Since $\delta V_{H}>\delta V_{L}$, a strategy would be weakly dominated if the bid at $t=0$ is different from the willingness to pay for obtaining $\delta V_{H}$ instead of $\delta V_{L}$. This is against the assumption that $x_{H}^{*}<x_{L}^{*}$.

Finally, suppose that $x_{H}^{*}=x_{L}^{*}$. This would lead to assigning victory at random in each time. However, a slightly higher bid would allow winning the competition for sure, determining a gain of expected utility. As a consequence, the profile under consideration cannot even be a Nash equilibrium.

Proof of Proposition 2

Proof. We focus on the relationship between $x^{*}$ and $R_{L}$, since we can then conclude on $I$ by noting that $I$ is uniquely and inversely related with $R_{L}$, due to $I=R-2 R_{L}$. By looking at (2) and using the implicit function theorem, there exists a differentiable function $x^{*}\left(R_{L}\right)$ for $R_{L} \in(0, R / 2)$, whose change in response to a marginal redistribution is:

$$
\frac{\mathrm{d} x^{*}}{\mathrm{~d} R_{L}}=\frac{U^{\prime}\left(R_{L}-x^{*}\right)-\frac{\delta}{1-\delta} U^{\prime}\left(R_{H}-x^{*}\right)-\frac{1}{1-\delta} U^{\prime}\left(R_{L}\right)}{U^{\prime}\left(R_{L}-x^{*}\right)+\frac{\delta}{1-\delta} U^{\prime}\left(R_{H}-x^{*}\right)} .
$$

We now prove the second part of the statement. It is immediate to recognize that $x^{*}=0$ solves (2) when $R_{L}=R_{H}$. By the continuity of $x^{*}\left(R_{L}\right)$, this shows that $\lim _{R_{L} \rightarrow R / 2} x^{*}\left(R_{L}\right)=0$. Since $x^{*}$ is bounded from above by $R_{L}$, we can also conclude that $\lim _{R_{L} \rightarrow 0} x^{*}\left(R_{L}\right)=0$, since $R_{L}$ tends to 0 when $I$ tends to $R$.

Finally we prove the existence of the threshold $\hat{I}$. Since $x^{*}\left(R_{L}\right)$ is differentiable, and tends to 0 when $R_{L}$ tends both to 0 and to $R / 2$, we know that there must exist $\hat{R}_{L}$ (and hence $\left.\hat{I}\right)$ such that $\left(\mathrm{d} x^{*} / \mathrm{d} R_{L}\right)\left(\hat{R_{L}}\right)=0$. It still remains to prove that $x^{*}\left(R_{L}\right)$ is increasing for $R_{L}>\hat{R_{L}}$ (hence increasing for $I<\hat{I}$ ) and decreasing for $R_{L}<\hat{R}_{L}$ (hence decreasing for $I>\hat{I}$ ). For such a purpose, it is enough to take the second order derivative at such $\hat{R}_{L}$, and show that it must be negative, so that $\hat{R}_{L}$ is the unique point where the derivative is null; indeed, if the first derivative were null at more than one point, the second derivative should be allowed to be positive at some of these points. We observe that $\left(\mathrm{d}^{2} x^{*} / \mathrm{d} R_{L}^{2}\right)\left(\hat{R_{L}}\right)<0$ if and only if the following inequality - obtained by exploiting that $\left(\mathrm{d} x^{*} / \mathrm{d} R_{L}\right)\left(\hat{R_{L}}\right)=0$ and that the denominator of $(14)$ is always positive is satisfied:

$$
U^{\prime \prime}\left(R_{L}-x^{*}\right)+\frac{\delta}{1-\delta} U^{\prime \prime}\left(R_{H}-x^{*}\right)-\frac{1}{1-\delta} U^{\prime \prime}\left(R_{L}\right)<0 .
$$

The assumption that the coefficient of absolute risk aversion is never increasing implies that:

$$
-\frac{U^{\prime \prime}\left(R_{L}-x^{*}\right)}{U^{\prime}\left(R_{L}-x^{*}\right)} \geq-\frac{U^{\prime \prime}\left(R_{L}\right)}{U^{\prime}\left(R_{L}\right)} .
$$

If we multiply both sides by $-\left[U^{\prime}\left(R_{L}\right) U^{\prime}\left(R_{L}-x^{*}\right)\right] /(1-\delta)$, and reversing the inequality, we obtain:

$$
\frac{1}{1-\delta} U^{\prime}\left(R_{L}\right) U^{\prime \prime}\left(R_{L}-x^{*}\right) \leq \frac{1}{1-\delta} U^{\prime}\left(R_{L}-x^{*}\right) U^{\prime \prime}\left(R_{L}\right) \text {. }
$$

Since we are taking this second derivative at a point where the first derivative is null, we recognize from (14) that $U^{\prime}\left(R_{L}-x^{*}\right)>[1 /(1-\delta)] U^{\prime}\left(R_{L}\right)$, which allows us to derive from (17) the following inequality:

$$
U^{\prime \prime}\left(R_{L}-x^{*}\right)<\frac{1}{1-\delta} U^{\prime \prime}\left(R_{L}\right)
$$

We can obtain (15) from (18) by moving to the left-hand side the term which is in the right-hand side, and adding the negative term $[\delta /(1-\delta)] U^{\prime \prime}\left(R_{H}-x^{*}\right)$.

Proof of Proposition 3

Proof. When the degree of inequality tends to $R$, then $W$ tends to $-\infty$, because $R_{L}$ tends to 0 so that $U\left(R_{L}\right)$ tends to $-\infty$. Moreover, $W$ is always decreasing in the range $(0, \hat{I}]$, since an increase in $I$ determines both an increase in $x^{*}$ and a more unequal distribution of resources. Finally, we note that, if a local maximum of $W$ exists in $(\hat{I}, R)$, then we can find another level of inequality in $(0, \hat{I})$ where $x^{*}$ is the same, and hence the social welfare is strictly larger (due to the more equal distribution of resources).

Proof of Proposition 4

Proof. We fix $I \in(0, R)$. By the implicit function theorem we know that $x^{*}$ can be seen as a differentiable function of $\delta$, for $\delta \in(0,1)$. In particular, the derivative turns out to be

$$
\frac{\mathrm{d} x^{*}}{\mathrm{~d} \delta}=\frac{U\left(R_{H}-x^{*}\right)-U\left(R_{L}\right)}{(1-\delta)^{2} U^{\prime}\left(R_{L}-x^{*}\right)+\delta(1-\delta) U^{\prime}\left(R_{H}-x^{*}\right)},
$$

which is always positive. 


\section{Proof of Proposition 5}

Preliminarily, let us define the following implicit functions from Eqs. (8) and (9):

$$
\begin{aligned}
f_{0}\left(R_{L}^{y}, R_{L}^{o}, p\right)= & U\left(R_{L}^{o}-x_{o}^{*}\right)+\frac{(1-p) \delta}{1-(1-p) \delta^{2}}\left[U\left(R_{H}^{y}-x_{y}^{*}\right)+\delta U\left(R_{H}^{o}-x_{o}^{*}\right)\right] \\
& -U\left(R_{L}^{o}\right)-\frac{(1-p) \delta}{1-(1-p) \delta^{2}}\left[U\left(R_{L}^{y}\right)+\delta U\left(R_{L}^{o}\right)\right]=0 ; \\
f_{y}\left(R_{L}^{y}, R_{L}^{o}, p\right)= & U\left(R_{L}^{y}-x_{y}^{*}\right)+\delta U\left(R_{H}^{o}-x_{o}^{*}\right)+\frac{(1-p) \delta^{2}}{1-(1-p) \delta^{2}}\left[U\left(R_{H}^{y}-x_{y}^{*}\right)+\delta U\left(R_{H}^{o}-x_{o}^{*}\right)\right] \\
& -U\left(R_{L}^{y}\right)-\delta U\left(R_{L}^{o}\right)-\frac{(1-p) \delta^{2}}{1-(1-p) \delta^{2}}\left[U\left(R_{L}^{y}\right)+\delta U\left(R_{L}^{o}\right)\right]=0 .
\end{aligned}
$$

In the following we provide two lemmas that are then applied in this proof and in the proof of Proposition 6.

Lemma 1. There exists a unique pair of $x_{y}^{*}$ and $x_{0}^{*}$ that jointly satisfies $f_{o}\left(R_{L}^{y}, R_{L}^{o}, p\right)=0$ and $f_{y}\left(R_{L}^{y}, R_{L}^{o}, p\right)=0$.

Proof. Taking the total differential of $f_{o}\left(R_{L}^{y}, R_{L}^{o}, p\right)$ and $f_{y}\left(R_{L}^{y}, R_{L}^{o}, p\right)$ with respect to $x_{y}^{*}$ and $x_{o}^{*}$ we get, respectively:

$$
\begin{aligned}
& \frac{\mathrm{d} x_{o}^{*}}{\mathrm{~d} x_{y}^{*}}=-\frac{\frac{(1-p) \delta}{1-(1-p) \delta^{2}} U^{\prime}\left(R_{H}^{y}-x_{y}^{*}\right)}{U^{\prime}\left(R_{L}^{o}-x_{o}^{*}\right)+\delta U^{\prime}\left(R_{H}^{o}-x_{o}^{*}\right) \frac{(1-p) \delta}{1-(1-p) \delta^{2}}} ; \\
& \frac{\mathrm{d} x_{o}^{*}}{\mathrm{~d} x_{y}^{*}}=-\frac{\frac{(1-p) \delta}{1-(1-p) \delta^{2}} U^{\prime}\left(R_{H}^{y}-x_{y}^{*}\right)+\frac{1}{\delta} U^{\prime}\left(R_{L}^{y}-x_{y}^{*}\right)}{U^{\prime}\left(R_{H}^{o}-x_{o}^{*}\right)+\delta U^{\prime}\left(R_{H}^{o}-x_{o}^{*}\right) \frac{(1-p) \delta}{1-(1-p) \delta^{2}}} .
\end{aligned}
$$

Both (22) and (23) are strictly negative, with (23) being always larger than (22) in absolute value. Hence, the graphs of $f_{o}\left(R_{L}^{y}, R_{L}^{o}, p\right)$ and $f_{y}\left(R_{L}^{y}, R_{L}^{o}, p\right)$ can cross at most once in the $\left(x_{y}^{*}, x_{0}^{*}\right)$-plane. Moreover, we have that when $x_{y}^{*}=0$ Eqs. (20) and (21) become:

$$
\begin{aligned}
& \frac{(1-p) \delta}{1-(1-p) \delta^{2}}\left[U\left(R_{H}^{y}\right)+\delta U\left(R_{H}^{o}-x_{o}^{*}\right)-U\left(R_{L}^{y}\right)-\delta U\left(R_{L}^{o}\right)\right]+U\left(R_{L}^{o}-x_{o}^{*}\right)-U\left(R_{L}^{o}\right)=0 \\
& \frac{(1-p) \delta^{2}}{1-(1-p) \delta^{2}}\left[U\left(R_{H}^{y}\right)+\delta U\left(R_{H}^{o}-x_{o}^{*}\right)-U\left(R_{L}^{y}\right)-\delta U\left(R_{L}^{o}\right)\right]+\delta U\left(R_{H}^{o}-x_{o}^{*}\right)-\delta U\left(R_{L}^{o}\right)=0
\end{aligned}
$$

while when $x_{0}^{*}=0$ Eqs. (20) and (21) become:

$$
\begin{aligned}
& \frac{(1-p) \delta}{1-(1-p) \delta^{2}}\left[U\left(R_{H}^{y}-x_{y}^{*}\right)+\delta U\left(R_{H}^{o}\right)-U\left(R_{L}^{y}\right)-\delta U\left(R_{L}^{o}\right)\right]=0 ; \\
& \frac{(1-p) \delta^{2}}{1-(1-p) \delta^{2}}\left[U\left(R_{H}^{y}-x_{y}^{*}\right)+\delta U\left(R_{H}^{o}\right)-U\left(R_{L}^{y}\right)-\delta U\left(R_{L}^{o}\right)\right]+U\left(R_{L}^{y}-x_{y}^{*}\right)+\delta U\left(R_{H}^{o}\right)-U\left(R_{L}^{y}\right)-\delta U\left(R_{L}^{o}\right)=0 .
\end{aligned}
$$

By direct inspection of (24) and (25), we see that (24) is satisfied for a value of $x_{0}^{*}$ which is strictly smaller than the one that satisfies (25), since $U\left(R_{H}^{o}\right)>U\left(R_{L}^{o}\right)$. Moreover, (24) implies that $x_{y}^{*}$ must be such that $U\left(R_{H}^{y}-x_{y}^{*}\right)+\delta U\left(R_{H}^{o}\right)=U\left(R_{L}^{y}\right)+$ $\delta U\left(R_{L}^{o}\right)$, and hence that for this same value (27) is strictly negative since $U\left(R_{L}^{y}\right)<U\left(R_{H}^{y}\right)$, which in turn implies that (26) is satisfied for a value of $x_{y}^{*}$ which is strictly larger than the one that satisfies (27). Therefore, the graphs of $f_{o}\left(R_{L}^{y}, R_{L}^{o}, p\right)$ and $f_{y}\left(R_{L}^{y}, R_{L}^{o}, p\right)$ cross just once and this happens in the region where $x_{y}^{*}>0$ and $x_{o}^{*}>0$.

Lemma 2. The derivatives of $x_{y}^{*}$ and $x_{0}^{*}$ with respect to $\gamma \in\left\{R_{L}^{y}, R_{L}^{o}, p\right\}$ are given by:

$$
\begin{gathered}
\frac{\mathrm{d} x_{y}^{*}}{\mathrm{~d} \gamma}=\frac{\frac{\partial f_{y}}{\partial x_{o}^{*}} \frac{\partial f_{o}}{\partial \gamma}-\frac{\partial f_{o}}{\partial x_{o}^{*}} \frac{\partial f_{y}}{\partial \gamma}}{\frac{\partial f_{y}}{\partial x_{y}^{*}} \frac{\partial f_{o}}{\partial x_{o}^{*}}-\frac{\partial f_{o}}{\partial x_{y}^{*}} \frac{\partial f_{y}^{*}}{\partial x_{o}^{*}}} ; \\
\frac{\mathrm{d} x_{o}^{*}}{\mathrm{~d} \gamma}=\frac{\frac{\partial f_{o}}{\partial x_{y}^{*}} \frac{\partial f_{y}}{\partial \gamma}-\frac{\partial f_{y}}{\partial x_{o}^{*}} \frac{\partial f_{y}}{\partial \gamma}}{\frac{\partial f_{y}}{\partial x_{y}^{*}} \frac{\partial f_{o}}{\partial x_{o}^{*}}-\frac{\partial f_{o}}{\partial x_{y}^{*}} \frac{\partial f_{y}^{*}}{\partial x_{o}^{*}}} ;
\end{gathered}
$$

with $\frac{\partial f_{y}}{\partial x_{0}^{*}} \frac{\partial f_{o}}{\partial x_{o}^{*}}-\frac{\partial f_{o}}{\partial x_{y}^{*}} \frac{\partial f_{y}}{\partial x_{0}^{*}}>0$. 
Proof. Derivatives (28) and (29) follow from the application of the Implicit Function Theorem to Eqs. (20) and (21) to get the local value of the derivative of $x_{y}^{*}$ and of $x_{0}^{*}$ with respect to $\gamma$, which gives:

$$
\left[\begin{array}{l}
\mathrm{d} x_{y}^{*} \\
\mathrm{~d} \gamma \\
\frac{\mathrm{d} x_{0}^{*}}{\mathrm{~d} \gamma}
\end{array}\right]=-\left[\begin{array}{ll}
\frac{\partial f_{o}}{\partial x_{y}^{*}} & \frac{\partial f_{o}}{\partial x_{o}^{*}} \\
\frac{\partial f_{y}^{*}}{\partial x_{y}^{*}} & \frac{\partial f_{y}}{\partial x_{o}^{*}}
\end{array}\right]^{-1}\left[\begin{array}{l}
\frac{\mathrm{d} f_{y}}{\mathrm{~d} \gamma} \\
\frac{\mathrm{d} f_{o}}{\mathrm{~d} \gamma}
\end{array}\right] .
$$

The preceding expression can be transformed into:

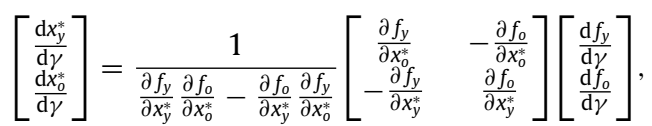

from which (28) and (29) can be directly calculated.

The remaining part of the lemma follows from the fact that

$$
\left|\frac{\partial f_{o}}{\partial x_{o}^{*}}\right|>\delta\left|\frac{\partial f_{y}}{\partial x_{o}^{*}}\right| \text { and } \delta\left|\frac{\partial f_{y}}{\partial x_{y}^{*}}\right|>\left|\frac{\partial f_{o}}{\partial x_{y}^{*}}\right|
$$

that in turn is obtained from direct inspection of the following derivatives:

$$
\begin{aligned}
& \frac{\partial f_{o}}{\partial x_{o}^{*}}=-U^{\prime}\left(R_{L}^{o}-x_{o}^{*}\right)-\frac{(1-p) \delta^{2}}{1-(1-p) \delta^{2}} U^{\prime}\left(R_{H}^{o}-x_{o}^{*}\right) ; \\
& \frac{\partial f_{y}}{\partial x_{o}^{*}}=-\delta U^{\prime}\left(R_{H}^{o}-x_{o}^{*}\right)-\frac{(1-p) \delta^{3}}{1-(1-p) \delta^{2}} U^{\prime}\left(R_{H}^{o}-x_{o}^{*}\right) ; \\
& \frac{\partial f_{y}}{\partial x_{y}^{*}}=-U^{\prime}\left(R_{L}^{y}-x_{y}^{*}\right)-\frac{(1-p) \delta^{2}}{1-(1-p) \delta^{2}} U^{\prime}\left(R_{H}^{y}-x_{y}^{*}\right) ; \\
& \frac{\partial f_{o}}{\partial x_{y}^{*}}=-\frac{(1-p) \delta}{1-(1-p) \delta^{2}} U^{\prime}\left(R_{H}^{y}-x_{y}^{*}\right) .
\end{aligned}
$$

We are now ready to provide the proof of Proposition 5.

From Lemma 1 we have that there exists a unique pair of $x_{y}^{*}$ and $x_{0}^{*}$ that jointly satisfies $f_{o}\left(R_{L}^{y}, R_{L}^{o}, p\right)=0$ and $f_{y}\left(R_{L}^{y}, R_{L}^{o}, p\right)=0$. This in turn implies that also the following conditions are satisfied uniquely by some bids $x_{o H}^{*}>x_{o}^{*}$ and $x_{o H}^{*}>x_{O}^{*}$ made by the agent who is currently $H$ when young:

$$
\begin{gathered}
U\left(R_{H}^{o}-x_{o H}^{*}\right)+\frac{(1-p) \delta}{1-(1-p) \delta^{2}}\left[U\left(R_{H}^{y}-x_{y L}^{*}\right)+\delta U\left(R_{H}^{o}-x_{o L}^{*}\right)\right]=U\left(R_{H}^{o}\right)+\frac{(1-p) \delta}{1-(1-p) \delta}\left[U\left(R_{L}^{y}\right)+\delta U\left(R_{L}^{o}\right)\right] \\
U\left(R_{H}^{y}-x_{y H}^{*}\right)+ \\
\delta U\left(R_{H}^{o}-x_{o L}^{*}\right)+\frac{(1-p) \delta^{2}}{1-(1-p) \delta^{2}}\left[U\left(R_{H}^{y}-x_{y L}^{*}\right)+\delta U\left(R_{H}^{o}-x_{o L)}^{*}\right]\right. \\
=U\left(R_{H}^{y}\right)+\delta U\left(R_{L}^{o}\right)+\frac{(1-p) \delta^{2}}{1-(1-p) \delta}\left[U\left(R_{L}^{y}\right)+\delta U\left(R_{L}^{o}\right)\right] .
\end{gathered}
$$

By Lemma 2, setting $\gamma=R_{L}^{y}$ and $\gamma=R_{L}^{o}$ we get, respectively:

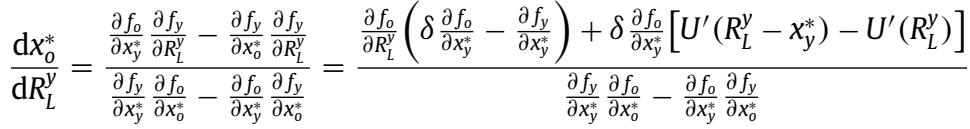

$$
\begin{aligned}
& \frac{\mathrm{d} x_{y}^{*}}{\mathrm{~d} R_{L}^{o}}=\frac{\frac{\partial f_{y}}{\partial x_{0}^{*}} \frac{\partial f_{o}}{\partial R_{L}^{o}}-\frac{\partial f_{o}}{\partial x_{o}^{*}} \frac{\partial f_{y}}{\partial R_{L}^{o}}}{\frac{\partial f_{y}}{\partial x_{y}^{*}} \frac{\partial f_{o}}{\partial x_{o}^{*}}-\frac{\partial f_{0}}{\partial x_{y}^{*}} \frac{\partial f_{y}}{\partial x_{o}^{*}}}=\frac{\frac{\partial f_{y}}{\partial R_{L}^{o}}\left(\frac{1}{\delta} \frac{\partial f_{y}}{\partial x_{o}^{*}}-\frac{\partial f_{o}}{\partial x_{o}^{*}}\right)+\frac{1}{\delta} \frac{\partial f_{y}}{\partial x_{o}^{*}}\left[U^{\prime}\left(R_{L}^{o}-x_{o}^{*}\right)+U^{\prime}\left(R_{L}^{o}-x_{o}^{*}\right)\right]}{\frac{\partial f_{y}}{\partial x_{y}^{*}} \frac{\partial f_{o}}{\partial x_{o}^{*}}-\frac{\partial f_{0}}{\partial x_{y}^{*}} \partial f_{y}} \partial
\end{aligned}
$$

where the second equality in (37) follows from:

$$
\begin{aligned}
& \frac{\partial f_{o}}{\partial x_{y}^{*}}=-\frac{(1-p) \delta}{1-(1-p) \delta^{2}}\left[U^{\prime}\left(R_{H}^{y}-x_{y}^{*}\right)+U^{\prime}\left(R_{L}^{y}\right)\right] \\
& \frac{\partial f_{y}}{\partial x_{y}^{*}}=U^{\prime}\left(R_{L}^{y}-x_{y}^{*}\right)-U^{\prime}\left(R_{L}^{y}\right)-\frac{(1-p) \delta^{2}}{1-(1-p) \delta^{2}}\left[U^{\prime}\left(R_{H}^{y}-x_{y}^{*}\right)+U^{\prime}\left(R_{L}^{y}\right)\right]
\end{aligned}
$$


which implies that:

$$
\frac{\partial f_{1}}{\partial x_{y}^{*}}=U^{\prime}\left(R_{L}^{y}-x_{y}^{*}\right)-U^{\prime}\left(R_{L}^{y}\right)+\delta \frac{\partial f_{o}}{\partial x_{y}^{*}}
$$

while the second equality in (38) follows from:

$$
\begin{aligned}
& \frac{\partial f_{o}}{\partial x_{o}^{*}}=U^{\prime}\left(R_{L}^{o}-x_{o}^{*}\right) U^{\prime}\left(R_{L}^{o}\right)-\frac{(1-p) \delta^{2}}{1-(1-p) \delta^{2}}\left[U^{\prime}\left(R_{H}^{o}-x_{o}^{*}\right)+U^{\prime}\left(R_{L}^{o}\right)\right] \\
& \frac{\partial f_{y}}{\partial x_{o}^{*}}=-\delta U^{\prime}\left(R_{H}^{o}-x_{o}^{*}\right)-\delta U^{\prime}\left(R_{L}^{o}\right)-\frac{(1-p) \delta^{3}}{1-(1-p) \delta^{2}}\left[U^{\prime}\left(R_{H}^{o}-x_{o}^{*}\right)+U^{\prime}\left(R_{L}^{o}\right)\right] ;
\end{aligned}
$$

which implies that:

$$
\frac{\partial f_{o}}{\partial x_{o}^{*}}=U^{\prime}\left(R_{L}^{o}-x_{o}^{*}\right)+U^{\prime}\left(R_{H}^{o}-x_{o}^{*}\right)+\frac{1}{\delta} \frac{\partial f_{1}}{\partial x_{o}^{*}} .
$$

To establish that (38) is negative it is enough to note that, by Lemma 2, the denominator is strictly positive and, by (30), $\left(\frac{\partial f_{y}}{\partial x_{o}^{*}}-\delta \frac{\partial f_{o}}{\partial x_{o}^{*}}\right)>0$, since $\frac{\partial f_{o}}{\partial R_{L}^{y}}<0$ and $\frac{\partial f_{o}}{\partial x_{o}^{*}}<0$.

Similarly, to establish that (37) is negative it is enough to note that, by Lemma 2, the denominator is strictly positive and, by (30), $\left(\frac{\partial f_{0}}{\partial x_{y}^{*}}-\frac{1}{\delta} \frac{\partial f_{o} y}{\partial x_{y}^{*}}\right)>0$, since $\frac{\partial f_{y}}{\partial R_{L}^{0}}<0$ and $\frac{\partial f_{y}}{\partial x_{y}^{*}}<0$.

\section{Proof of Proposition 6}

We follow the arguments used in the proof of Proposition 5, and by setting $\gamma=p$ we get from Lemma 2:

$$
\begin{aligned}
& \frac{\mathrm{d} x_{y}^{*}}{\mathrm{~d} p}=\frac{\frac{\partial f_{y}}{\partial x_{o}^{*}} \frac{\partial f_{o}}{\partial p}-\frac{\partial f_{o}}{\partial x_{o}^{*}} \frac{\partial f_{y}}{\partial p}}{\frac{\partial y_{y}}{\partial x_{y}^{*}} \frac{\partial f_{o}}{\partial x_{o}^{*}}-\frac{\partial f_{o}}{\partial x_{y}^{*}} \frac{\partial f_{y}}{\partial x_{o}^{*}}}=\frac{\frac{\partial f_{o}}{\partial p}\left(\frac{\partial f_{y}}{\partial x_{o}^{*}}-\delta \frac{\partial f_{o}}{\partial x_{o}^{*}}\right)}{\frac{\partial f_{y}}{\partial x_{y}^{*}} \frac{\partial f_{o}}{\partial x_{o}^{*}}-\frac{\partial f_{o}}{\partial x_{y}^{*}} \frac{\partial f_{y}}{\partial x_{o}^{*}}} ; \\
& \frac{\mathrm{d} x_{0}^{*}}{\mathrm{~d} p}=\frac{\frac{\partial f_{0}}{\partial x_{y}^{*}} \frac{\partial f_{y}}{\partial p}-\frac{\partial f_{y}}{\partial x_{o}^{*}} \frac{\partial f_{y}}{\partial p}}{\frac{\partial y_{y}}{\partial x_{y}^{*}} \frac{\partial f_{o}}{\partial x_{o}^{*}}-\frac{\partial f_{o}}{\partial x_{y}^{*}} \frac{\partial f_{y}}{\partial x_{0}^{*}}}=\frac{\frac{\partial f_{o}}{\partial p}\left(\delta \frac{\partial f_{o}}{\partial x_{y}^{*}}-\frac{\partial f_{y}}{\partial x_{y}^{*}}\right)}{\frac{\partial f_{y}}{\partial x_{y}^{*}} \frac{\partial f_{o}}{\partial x_{o}^{*}}-\frac{\partial f_{o}}{\partial x_{y}^{*}} \frac{\partial f_{y}}{\partial x_{o}^{*}}} ;
\end{aligned}
$$

where the second equality in (45) and (46) follows from:

$$
\begin{aligned}
& \frac{\partial f_{o}}{\partial p}=-\frac{1-(1-p) \delta^{2}(1-\delta)}{\left[1-(1-p) \delta^{2}\right]^{2}}\left[U^{\prime}\left(R_{H}^{y}-x_{y}^{*}\right)-U^{\prime}\left(R_{L}^{y}\right)+U^{\prime}\left(R_{H}^{o}-x_{o}^{*}\right)-U^{\prime}\left(R_{L}^{o}\right)\right] \\
& \frac{\partial f_{y}}{\partial p}=-\frac{1-(1-p) \delta^{3}(1-\delta)}{\left[1-(1-p) \delta^{2}\right]^{2}}\left[U^{\prime}\left(R_{H}^{y}-x_{y}^{*}\right)-U^{\prime}\left(R_{L}^{y}\right)+U^{\prime}\left(R_{H}^{o}-x_{o}^{*}\right)-U^{\prime}\left(R_{L}^{o}\right)\right]
\end{aligned}
$$

which implies that:

$$
\frac{\partial f_{1}}{\partial p}=\delta \frac{\partial f_{o}}{\partial p}
$$

To establish that (45) and (46) are negative it is enough to note that, by Lemma 2, the denominator is strictly positive and, by (30), $\left(\frac{\partial f_{y}}{\partial x_{0}^{*}}-\delta \frac{\partial f_{o}}{\partial x_{o}^{*}}\right)>0$, since $\frac{\partial f_{o}}{\partial p}<0$.

\section{Proof of Proposition 7}

Proof. Preliminarily, we study the derivative of $x^{*}$ with respect to $R_{L}$ when $R_{H}$ is kept fixed. We call such a function $\tilde{x}^{*}\left(R_{L}\right)$, with $R_{L} \in\left(0, R_{H}\right)$, to distinguish it from the case when an increase of $R_{L}$ implies a reduction of $R_{H}$. Such a function exists and it is differentiable by virtue of the implicit function theorem applied to (2). Hence:

$$
\frac{\mathrm{d} \tilde{x}^{*}}{\mathrm{~d} R_{L}}=\frac{U^{\prime}\left(R_{L}-\tilde{x}^{*}\right)-\frac{1}{1-\delta} U^{\prime}\left(R_{L}\right)}{U^{\prime}\left(R_{L}-\tilde{x}^{*}\right)+\frac{\delta}{1-\delta} U^{\prime}\left(R_{H}-\tilde{x}^{*}\right)} .
$$

Recall that $x^{*}=0$ solves (2) when $R_{L}=R_{H}$. By the continuity of $\tilde{x}^{*}\left(R_{L}\right)$, this shows that $\lim _{R_{L} \rightarrow R_{H}} \tilde{x}^{*}\left(R_{L}\right)=0$. Since $x^{*}$ is bounded from above by $R_{L}$, we can also conclude that $\lim _{R_{L} \rightarrow 0} \tilde{x}^{*}\left(R_{L}\right)=0$. 
Since $\tilde{x}^{*}\left(R_{L}\right)$ is differentiable, there must exist $\tilde{R}_{L}$ such that (50) is equal to 0 . To show that $\mathrm{d} x^{*} / \mathrm{d} R_{L}$ is positive for $R_{L}>\tilde{R}_{L}$ and negative for $R_{L}<\tilde{R}_{L}$ it suffices to show that the second order derivative at $\tilde{R}_{L}$ is negative (see proof of Proposition 2 ). Such second derivative is non-positive if and only if the following inequality is satisfied:

$$
U^{\prime \prime}\left(R_{L}-\tilde{x}^{*}\right)-\frac{1}{1-\delta} U^{\prime \prime}\left(R_{L}\right) \leq 0 .
$$

Recall that from the assumption of non-decreasing absolute risk aversion (see proof of Proposition 2) we have:

$$
\frac{1}{1-\delta} U^{\prime}\left(R_{L}\right) U^{\prime \prime}\left(R_{L}-\tilde{x}^{*}\right) \leq \frac{1}{1-\delta} U^{\prime}\left(R_{L}-\tilde{x}^{*}\right) U^{\prime \prime}\left(R_{L}\right)
$$

Since the first derivative is null at $\tilde{R}_{L}$, from (50) we obtain that $U^{\prime}\left(\tilde{R}_{L}-\tilde{x}^{*}\right)=[1 /(1-\delta)] U^{\prime}\left(\tilde{R}_{L}\right)$, which implies that (51) holds.

We now prove that $\tilde{R}_{L}$ is unique. Suppose not and let $\tilde{R}_{L}$ and $\tilde{R}_{L}^{\prime}$ be distinct values such that (50) is zero. Then, by (51), we get $\tilde{x}^{*}\left(\tilde{R}_{L}\right)=\tilde{x}^{*}\left(\tilde{R}_{L}^{\prime}\right)$. However, in the light of (2), this is impossible since the two sides of (2) increase at different rates in $R_{L}$ due to the strict concavity of $U$ which implies that, for a fixed $x^{*}, U^{\prime}\left(R_{L}-x^{*}\right)=[1 /(1-\delta)] U^{\prime}\left(R_{L}\right)$ at most for a single value of $R_{L}$.

Finally, we take the derivative of $S_{H}$ with respect to $R_{L}$ keeping $R_{H}$ fixed and the derivative of $S_{L}$ with respect $R_{H}$ keeping $R_{L}$ fixed:

$$
\begin{aligned}
\frac{\mathrm{d} S_{H}}{\mathrm{~d} R_{L}} & =-\sum_{t=1}^{\infty} \delta^{t} U^{\prime}\left(R_{H}-\tilde{x}^{*}\right) \frac{\mathrm{d} \tilde{x}^{*}}{\mathrm{~d} R_{L}}=-\sum_{t=1}^{\infty} \delta^{t} U^{\prime}\left(R_{H}-\tilde{x}^{*}\right) \frac{U^{\prime}\left(R_{L}-\tilde{x}^{*}\right)-\frac{1}{1-\delta} U^{\prime}\left(R_{L}\right)}{U^{\prime}\left(R_{L}-\tilde{x}^{*}\right)+\frac{\delta}{1-\delta} U^{\prime}\left(R_{H}-\tilde{x}^{*}\right)} ; \\
\frac{\mathrm{d} S_{L}}{\mathrm{~d} R_{H}} & =0 .
\end{aligned}
$$

By looking at (53) and (54), we complete the proof by observing that $\mathrm{d} x^{*} / \mathrm{d} R_{L}$ is positive in $\left(0, \tilde{R}_{L}\right)$ and negative in $\left(\tilde{R}_{L}, R_{H}\right)$.

\section{Appendix B. A signaling model of social competition}

In this appendix we provide and analyze a signaling variant of the second price auction model presented in the main text. All assumptions of the former model are retained, if not explicitly stated otherwise. Instead of two players, here we assume two player types with equal prior probability. We restrict attention to Markov strategies in this setting as well, with a Markov strategy mapping the amount of resources into a signal, i.e., $x:\left\{R_{H}, R_{L}\right\} \rightarrow \mathbb{R}_{+}$. This allows to maintain the notation where $x_{H}$ denotes the signal when $R_{H}$ resources are possessed, and $x_{L}$ denotes the signal when $R_{L}$ resources are possessed. However, differently from what happens to bids in the auction model, here signals do not compete with each other to earn the highest prize; rather, a signal generates a belief according to a function $\mu: \mathbb{R}_{+} \rightarrow[0,1]$, where $\mu(x)$ indicates the believed probability that signal $x$ comes from the player type with $R_{H}$ resources. By doing so, and consistently with the restriction to Markov strategies, we have imposed that beliefs are stationary over time. For analogous reasons, we look for equilibria that are robust to one-shot deviations only, i.e., changes in current signals in the assumption that future play remains unchanged. This allows to avoid embedding elements of cooperative analysis which would necessarily arise if the sender could plan her type-contingent future play. The assumption of one-shot deviations looks particularly reasonable if players at different time periods are interpreted as belonging to different generations.

As it is typically found in signaling games, here too there exists a multiplicity of pooling and separating equilibria, due to the great freedom of out-of-equilibrium beliefs. Proposition 8 states that, if we adopt a standard equilibrium refinement as the intuitive criterion (Cho and Kreps, 1987), then the amount of resources spent in signaling is the same as the price paid in the second price auction (see Proposition 1). Basically, the intuitive criterion asks that an out-of-equilibrium signal cannot be believed to come from a type of sender who would get surely a lower payoff from doing so with respect to the equilibrium payoff. This equilibrium refinement - as many other belief-based equilibrium refinements such as divinity and universal divinity (Banks and Sobel, 1987), D1 (Cho and Kreps, 1987), and stability (Kohlberg and Mertens, 1986) - selects the so-called Riley equilibrium (Riley, 2001), which in our case is the separating equilibrium where the type of sender with $R_{H}$ resources uses the cheapest signal that is unprofitable for the sender with $R_{L}$ resources to duplicate.

Given a profile of Markov strategies $\left(x_{H}, x_{L}\right)$ that is separating, i.e., such that $x_{H} \neq x_{L}$, we can compute the present discounted value of the overall stream of payoffs, denoted by $V_{H}$ for the player type with $R_{H}$ resources and by $V_{L}$ for the player type with $R_{L}$ resources:

$$
\begin{aligned}
& V_{H}=U\left(R_{H}-x_{H}\right)+\delta V_{H}, \\
& V_{L}=U\left(R_{L}-x_{L}\right)+\delta V_{L} ;
\end{aligned} \Longrightarrow \begin{aligned}
& V_{H}=\frac{U\left(R_{H}-x_{H}\right)}{1-\delta}, \\
& V_{L}=\frac{U\left(R_{L}-x_{L}\right)}{1-\delta} .
\end{aligned}
$$

We can do analogous computations when the profile of Markov strategies $\left(x_{H}, x_{L}\right)$ is pooling, i.e., such that $x_{H}=x_{L}$, obtaining:

$$
\begin{aligned}
& V_{H}=U\left(R_{H}-x_{H}\right)+\delta \frac{V_{H}+V_{L}}{2}, \\
& V_{L}=U\left(R_{L}-x_{H}\right)+\delta \frac{V_{H}+V_{L}}{2} ;
\end{aligned} \Longrightarrow \begin{aligned}
& V_{H}=U\left(R_{H}-x_{H}\right)+\delta \frac{U\left(R_{H}-x_{H}\right)+U\left(R_{L}-x_{H}\right)}{2(1-\delta)}, \\
& V_{L}=U\left(R_{L}-x_{H}\right)+\delta \frac{U\left(R_{H}-x_{H}\right)+U\left(R_{L}-x_{H}\right)}{2(1-\delta)}
\end{aligned}
$$


At any time period, the decision problem can hence be written as follows:

$$
\begin{array}{ll}
\max _{x^{\prime}} U\left(R_{H}-x^{\prime}\right)+\mu\left(x^{\prime}\right) \delta V_{H}+\left(1-\mu\left(x^{\prime}\right)\right) \delta V_{L} & \text { for the type with } R_{H} \text { resources, } \\
\max _{x^{\prime}} U\left(R_{L}-x^{\prime}\right)+\mu\left(x^{\prime}\right) \delta V_{H}+\left(1-\mu\left(x^{\prime}\right)\right) \delta V_{L} & \text { for the type with } R_{L} \text { resources. }
\end{array}
$$

The above formulation looks very similar to the decision problem of a standard signaling game. The only difference is given by the fact that the present values of the stream of payoffs, $V_{H}$ and $V_{L}$, depend on the profile of Markov strategies under consideration, as we can see from (55) and (56). We deal with this issue in the proof of Proposition 8.

Proposition 8. The unique symmetric Markov Perfect Equilibrium which survives the Intuitive Criterion is made of strategy $\left(x_{H}^{*}, x_{L}^{*}\right)$, where $x_{L}^{*}=0$ and $x_{H}^{*}$ is the unique solution to:

$$
U\left(R_{L}-x_{H}^{*}\right)+\sum_{t=1}^{\infty} \delta^{t} U\left(R_{H}-x_{H}^{*}\right)=U\left(R_{L}\right)+\sum_{t=1}^{\infty} \delta^{t} U\left(R_{L}\right) .
$$

Proof. In case of a pooling profile, we immediately recognize from (56) that $V_{H}>V_{L}$. In case of a separating profile, by looking at (55) we obtain that $V_{H}>V_{L}$ if and only if $x_{H}-x_{L}<R_{H}-R_{L}$. We note that when $x_{H}-x_{L} \geq R_{H}-R_{L}$, the profile under consideration violates individual rationality, since playing $x_{L}$ instead of $x_{H}$ would represent a profitable deviation for the type with $R_{H}$ resources. Since a Markov perfect equilibrium requires individual rationality of all decisions at any time period, we restrict attention to profiles where $V_{H}>V_{L}$.

If $V_{H}$ and $V_{L}$ were independent of $x_{H}$ and $x_{L}$, then we would have a standard signaling model where the intuition criterion is well known to select the profile where:

$$
\begin{aligned}
& U\left(R_{L}-x_{H}\right)+\delta V_{H}=U\left(R_{L}\right)+\delta V_{L}, \\
& x_{L}=0 .
\end{aligned}
$$

However, $V_{H}$ and $V_{L}$ do indeed depend on $x_{H}$ and $x_{L}$, as we can see by inspecting (55) and (56). Therefore, we have to require consistency by having (55) jointly satisfied with (58), which a strategy $\left(x_{H}^{*}, x_{L}^{*}\right)$, where $x_{L}^{*}=0$ and $x_{H}^{*}$ is solution to (57). This leads to identifying Markov perfect equilibria, because we are considering any Markov strategy which is individually rational and consistent at every time period, and which survives the intuitive criterion. Finally, we note that (57) has a unique solution, since the left-hand side is higher than the right-hand side when $x_{H}=0$, is lower when $x_{H}=R_{H}-R_{L}$, and continuously and monotonically decreases as $x_{H}$ increases, while the right-hand side is unaffected by $X_{H}$.

We note that, while equilibrium strategies in Proposition 8 differ from those in Proposition 1, an equivalence holds in terms of payments between the second price auction of the main text and the signaling variant considered in this appendix: the amount of resources spent in signaling is the same as the price paid in the second price auction.

\section{References}

Aronsson, T., Johansson-Stenman, O., 2010. Positional concerns in an olg model: optimal labor and capital income taxation. Int. Econ. Rev. 51 (4), 1071-1095. Aronsson, T., Johansson-Stenman, O., 2013. Conspicuous leisure: optimal income taxation when both relative consumption and relative leisure matter. Scand. J. Econ. 115 (1), 155-175

Banks, J.S., Sobel, J., 1987. Equilibrium selection in signaling games. Econometrica 647-661.

Bhaskar, V., Hopkins, E., 2016. Marriage as a rat race: noisy premarital investments with assortative matching. J. Polit. Econ. 124 (4), $992-1045$.

Bilancini, E., Boncinelli, L., 2012. Redistribution and the notion of social status. J. Public Econ. 96 (9), $651-657$.

Bilancini, E., Boncinelli, L., 2014. Instrumental cardinal concerns for social status in two-sided matching with non-transferable utility. Eur. Econ. Rev. 67, 174-189.

Bilancini, E., Boncinelli, L., 2019. Wage inequality, labor income taxes, and the notion of social status. Economics. 13 (2019-32), 1-35.

Cho, I.-K., Kreps, D.M., 1987. Signaling games and stable equilibria. Quart. J. Econ. 102 (2), 179-221.

Cole, H.L., Mailath, G.J., Postlewaite, A., 1992. Social norms, savings behavior, and growth. J. Polit. Econ. 100 (6), $1092-1125$.

Corneo, G., 2002. The efficient side of progressive income taxation. Eur. Econ. Rev. 46 (7), 1359-1368.

Corneo, G., Gruner, H.P., 2000. Social limits to redistribution. Am. Econ. Rev. 90 (5), 1491-1507.

Corneo, G., Jeanne, O., 1998. Social organization, status, and savings behavior. J. Public Econ. 70 (1), 37-51.

Corneo, G., Jeanne, O., 1999. Pecuniary emulation, inequality and growth. Eur. Eco.n Rev. 43 (9), 1665-1678.

Corneo, G., Jeanne, O., 2001. Status, the distribution of wealth, and growth. Scand. J. Econ. 103 (2), $283-293$.

Frank, R.H., 2008. Should public policy respond to positional externalities? J. Public Eco.n 92 (8), $1777-1786$.

Friend, I., Blume, M.E., 1975. The demand for risky assets. Am. Econ. Rev. 65 (5), 900-922.

Gallice, A., 2018. Social status, preferences for redistribution and optimal taxation: a survey. Economics 12 (2018-55), 1-17.

Gallice, A., Grillo, E., 2019. Economic and social-class voting in a model of redistribution with social concerns. J. Eur. Econ. Assoc..

Gallice, A., Grillo, E., 2019. A model of educational investment, social concerns, and inequality. Scand. J. Econ. 121 (4), $1620-1646$.

Hopkins, E., 2012. Job market signaling of relative position, or Becker married to Spence. J. Eur. Econ. Assoc. 10 (2), $290-322$.

Hopkins, E., Kornienko, T., 2004. Running to keep in the same place: consumer choice as a game of status. Am. Econ. Rev. 94 (4), $1085-1107$.

Hopkins, E., Kornienko, T., 2006. Inequality and growth in the presence of competition for status. Econ. Lett. 93 (2), 291-296.

Hopkins, E., Kornienko, T., 2010. Which inequality? The inequality of endowments versus the inequality of rewards. Am. Econ. Rev. 2 (3), 106-137.

Ireland, N.J., 1998. Status-seeking, income taxation and efficiency. J. Public Econ. 70 (1), 99-113.

Kohlberg, E., Mertens, J.-F., 1986. On the strategic stability of equilibria. Econometrica 1003-1037.

Levy, G., Razin, R., 2015. Preferences over equality in the presence of costly income sorting. Am. Econ. Rev. 7 (2), $308-337$

Maskin, E., Tirole, J., 2001. Markov perfect equilibrium: I. Observable actions. J. Econ. Theory 100 (2), $191-219$.

Postlewaite, A., 1998. The social basis of interdependent preferences. Eur. Econ. Rev. 42 (3), 779-800.

Riley, J.G., 2001. Silver signals: twenty-five years of screening and signaling. J. Econ. Lit. 39 (2), 432-478.

Truyts, T., 2010. Social status in economic theory. J. Econ. Surv. 24 (1), 137-169. 